\title{
Clinical Feasibility of Synthetic MRI in Multiple Sclerosis: A Diagnostic and Volumetric Validation Study
}

\author{
(D). Granberg, (DM. Uppman, (D). Hashim, (DC. Cananau, (D).E. Nordin, (D). Shams, (D). Berglund, (D). Forslin, (DP. Aspelin,
} (D) S. Fredrikson, and DM. Kristoffersen-Wiberg to-

\begin{abstract}
BACKGROUND AND PURPOSE: Quantitative MR imaging techniques are gaining interest as methods of reducing acquisition times while additionally providing robust measurements. This study aimed to implement a synthetic MR imaging method on a new scanner type and to compare its diagnostic accuracy and volumetry with conventional MR imaging in patients with MS and controls.
\end{abstract}

MATERIALS AND METHODS: Twenty patients with MS and 20 healthy controls were enrolled after ethics approval and written informed consent. Synthetic MR imaging was implemented on a Siemens 3T scanner. Comparable conventional and synthetic proton-density-, T1-, and T2-weighted, and FLAIR images were acquired. Diagnostic accuracy, lesion detection, and artifacts were assessed by blinded neuroradiologic evaluation, and contrast-to-noise ratios, by manual tracing. Volumetry was performed with synthetic MR imaging, FreeSurfer, FMRIB Software Library, and Statistical Parametric Mapping. Repeatability was quantified by using the coefficient of variance.

RESULTS: Synthetic proton-density-, T1-, and T2-weighted images were of sufficient or good quality and were acquired in 7\% less time than with conventional MR imaging. Synthetic FLAIR images were degraded by artifacts. Lesion counts and volumes were higher in synthetic MR imaging due to differences in the contrast of dirty-appearing WM but did not affect the radiologic diagnostic classification or lesion topography $(P=.50-.77)$. Synthetic MR imaging provided segmentations with the shortest processing time (16 seconds) and the lowest repeatability error for brain volume $(0.14 \%)$, intracranial volume $(0.12 \%)$, brain parenchymal fraction $(0.14 \%)$, and GM fraction (0.56\%).

CONCLUSIONS: Synthetic MR imaging can be an alternative to conventional MR imaging for generating diagnostic proton-density-, T1-, and T2-weighted images in patients with MS and controls while additionally delivering fast and robust volumetric measurements suitable for MS studies.

ABBREVIATIONS: $B P F=$ brain parenchymal fraction; $B V=$ brain volume; $\mathrm{CoV}=$ coefficient of variance; $\mathrm{GMF}=$ gray matter fraction; ICV = intracranial volume; $\mathrm{PD}=$ proton density; $\mathrm{SPM}=$ statistical parametric mapping; SyMRI = synthetic MR imaging; WMF = white matter fraction

In conventional MR imaging, multiple sequences with different contrast weightings are obtained. This process is time-consuming with redundant data acquisition. Techniques such as MR fingerprinting and synthetic MR imaging can reduce acquisition times and thereby increase MR imaging availability for both clin-

Received September 29, 2015; accepted after revision November 24.

From the Departments of Clinical Science, Intervention and Technology (T.G., M.U., F.H., L.E.N., S.S., J.B., Y.F., P.A., M.K.-W.) and Clinical Neuroscience (S.F.), Karolinska Institutet, Stockholm, Sweden; and Departments of Radiology (T.G., F.H., C.C., S.S., Y.F., P.A., M.K.-W), Diagnostic Medical Physics (M.U., L.E.N., J.B.), and Neurology (S.F.), Karolinska University Hospital, Stockholm, Sweden.

This work was supported by Karolinska Institutet and Stockholm County Council through an ALF grant.

Paper previously presented at: Annual Meeting of the Radiological Society of North America, November 29-December 1, 2015; Chicago, Illinois.

Please address correspondence to Tobias Granberg, MD, PhD, Department of Clinical Science, Intervention and Technology, Cl-46, Karolinska University Hospital, 141 86 Stockholm, Sweden; e-mail: tobias.granberg@ki.se ical applications and research. ${ }^{1-3}$ SyMRI is a synthetic MR imaging method based on a quantitative approach in which a single saturation recovery TSE sequence is used to estimate absolute physical properties, the proton density (PD), longitudinal relaxation rate, and transverse relaxation rate, including correction for $\mathrm{B}_{1}$-inhomogeneities. Rather than predetermining acquisition parameters such as TE, TI, and TR to maximize tissue contrast, ${ }^{3}$ synthetic MR imaging produces a free range of synthetic weightings based on a single sequence through mathematic inference. ${ }^{4,5}$ The quantitative nature of the method and its ability to probe multiple physical properties in a single sequence make it suitable for volumetric analysis. ${ }^{6-10}$ Synthetic MR imaging has shown

\footnotetext{
- Indicates open access to non-subscribers at www.ajnr.org

$\equiv$ Indicates article with supplemental on-line tables.

$\checkmark$ Indicates article with supplemental on-line photos.

http://dx.doi.org/10.3174/ajnr.A4665
}

AJNR Am J Neuroradiol 37:1023-29 Jun 2016 www.ajnr.org 
Table 1: MRI acquisition parameters

\begin{tabular}{|c|c|c|c|c|c|c|}
\hline Sequence Type & Synthetic MRI & TSE PD-/T2WI & FLAIR & TSE TIWI & 3D MPRAGE & 3D FLAIR \\
\hline Acquisition plane & Axial & Axial & Axial & Axial & Axial & Sagittal \\
\hline In-plane resolution (mm) & $0.9 \times 0.9$ & $0.9 \times 0.9$ & $0.9 \times 0.9$ & $0.9 \times 0.9$ & $0.9 \times 0.9$ & $1.0 \times 1.0$ \\
\hline Sections (No.) & 30 & 30 & 30 & 30 & 160 & 160 \\
\hline Section thickness (mm) & 4.0 & 4.0 & 4.0 & 4.0 & 1.0 & 1.0 \\
\hline Distance factor, (\%) & 30 & 30 & 30 & 30 & - & - \\
\hline Flip angle & $120^{\circ a}$ & $120^{\circ a}$ & $130^{\circ a}$ & $120^{\circ a}$ & $9^{\circ}$ & $120^{\circ}$ \\
\hline $\mathrm{TR}(\mathrm{ms})$ & $4260^{\mathrm{b}}$ & 4120 & 9000 & 550 & 1900 & 6000 \\
\hline TE (ms) & $22 / 100^{b}$ & $15 / 92$ & 90 & 8 & 3.48 & 388 \\
\hline TI (ms) & $150 / 580 / 2000 / 4130^{b}$ & - & 2500 & - & 900 & 2100 \\
\hline Turbo factor & 5 & 7 & 12 & 2 & - & - \\
\hline $\begin{array}{l}\text { Generalized partially parallel } \\
\text { acquisition factor }\end{array}$ & 2 & 0 & 2 & 0 & 2 & 2 \\
\hline Bandwidth (Hz/pixel) & 154 & 250 & 283 & 283 & 180 & 781 \\
\hline No. of averages & 1 & 1 & 1 & 2 & 1 & 1 \\
\hline Acquisition time & $6: 50$ & $2: 38$ & $3: 38$ & $4: 44$ & 5:15 & 7:02 \\
\hline
\end{tabular}

a Flip angles denote the flip angles of the echo pulses; excitation flip angles, $90^{\circ}$.

${ }^{\mathrm{b}}$ Acquisition parameters. Synthetic images were generated using TRs, TIs, and TEs matching the conventional sequences.

promising initial results for use in MS and patients with an ischemic event. ${ }^{11,12}$ The technique is consequently gaining interest as a potentially time-efficient alternative to conventional MR imaging to visualize and quantify brain tissue properties.

MS is a chronic neuroinflammatory disorder affecting 2.5 million people globally. ${ }^{13}$ MS has a heterogeneous clinical expression, which complicates the choice of disease-modifying therapy. ${ }^{14} \mathrm{MR}$ imaging is a cornerstone for the diagnosis and monitoring of MS, but qualitative MR imaging measurements are poorly correlated with the clinical outcome. Volumetric MR imaging measurements have an independent predictive value in MS but require laborious image postprocessing, limiting its clinical potential. ${ }^{15,16}$ Robust and fully automatic volumetry approved for clinical use would thus be important for clinical care and research purposes. The synthetic MR imaging technique has initially been developed for use on Philips (Best, the Netherlands) and GE Healthcare (Milwaukee, Wisconsin) MR imaging systems, but it is not available for other systems and independent evaluations of the method are scarce. ${ }^{5,6}$

The purpose of this study was to implement the synthetic MR imaging technique for use on Siemens (Erlangen, Germany) MR imaging scanners and to compare the diagnostic accuracy of synthetic and conventional images in MS. A secondary aim was to test the repeatability of the volumetric synthetic MR imaging measurements and compare the volumetric results and practicality with other commonly used brain volumetric methods.

\section{MATERIALS AND METHODS \\ Participants and Clinical Assessment}

In this prospective study, 20 patients with MS were recruited from the MS center at Karolinska University Hospital. A senior consultant in neurology (S.F.) performed neurologic examinations and rated physical disability by using the Expanded Disability Status Scale, blinded to the radiologic evaluations. Characteristics for patients with MS were the following: 16 women, 4 men; mean age, $44 \pm 14$ years. MS subtypes were 9 relapsing-remitting, $10 \mathrm{sec}-$ ondary-progressive, and 1 primary-progressive. The mean disease duration was $17.4 \pm 11$ years; median Expanded Disability Status
Scale score, 2.5 (range, 1.0-8.5) with disease-modifying therapy in 12 patients (60\%). A group of 20 age-matched neurologically healthy controls ( 11 women, 9 men; mean age, $41 \pm 17$ years) was also recruited. The ethics review board in Stockholm approved the study, and written informed consent was obtained from all participants.

\section{Image Acquisition}

Technical details for all MR imaging sequences are given in Table 1. Synthetic MR imaging was implemented as a saturation recovery TSE sequence with a saturation pulse flip angle of $120^{\circ}$, a multiecho readout including phase and magnitude data, with 4 repetitions on a Magnetom Trio 3T scanner (Siemens) on the VB17 software platform using a 12-channel head coil. The section acquisition order was altered for each repetition, resulting in 4 different effective saturation delays (150, 580,2000 , and $4130 \mathrm{~ms}$ ) for each section. The resulting data formed a matrix of 8 complex images per section at different saturation delays and TEs. A least squares fit was performed on the signal intensity of these images as previously described, ${ }^{3}$ resulting in estimates of the longitudinal and transverse relaxation rates, $\mathrm{PD}$, and $\mathrm{B}_{1}$ field inhomogeneities. The synthetic MR imaging acquisition time was 6:50 minutes. Conventional PD-, T1-, and T2-weighted, and FLAIR images were acquired on the basis of the standard brain imaging protocol of the clinic, with section thickness and in-plane resolutions matching those of the synthetic MR imaging sequence. Total acquisition time was 11:00 minutes for all $42 \mathrm{D}$ conventional MR imaging sequences. Conventional 3D T1WI MPRAGE and 3D FLAIR sequences were additionally acquired (12:17 minutes) for gold standard radiologic and volumetric analyses. Synthetic PD-, T1-, and T2-weighted, and FLAIR images were obtained by using synthetic MR imaging (SyMRI) 7.2 RC software (Synthetic MR, Linköping, Sweden) with synthetic TE, TI, and TR matching the conventional images. To study the repeatability of the volumetric SyMRI measurements, we temporarily took each participant out of the scanner and repositioned them for a second synthetic MR imaging acquisition. Similarly, a second MPRAGE sequence was obtained after re- 


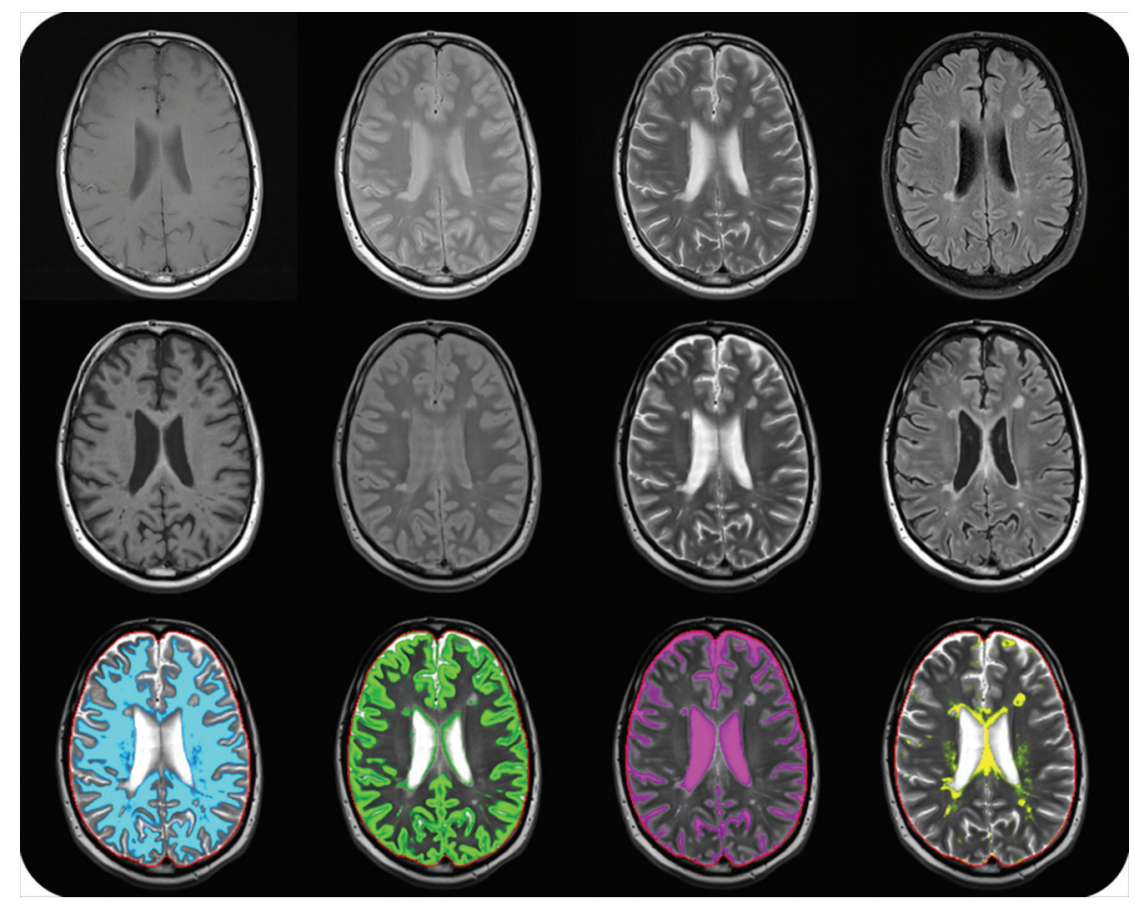

FIG 1. Conventional (top row) and synthetic (middle row) axial noncontrast MR imaging in a 49-year-old male patient with MS, from left to right: T1-, PD-, and T2-weighted, and FLAIR images. The bottom row shows brain tissue segmentations of the WM (cyan), GM (green), CSF (magenta), and other remaining brain tissues (yellow) from SyMRI.

positioning in 5 controls, to compare the repeatability of conventional and synthetic volumetrics.

\section{Radiologic Assessment}

All images were blindly and independently assessed by a neuroradiologist and a fellow in neuroradiology (F.H., C.C.) on standard radiologic workstations in the PACS in a random order in 2 sessions 2-4 weeks apart, in which each participant was only represented once per session with either the synthetic or conventional images. Image quality was rated on a 4-level scale: poor (artifacts or image-quality issues rendering the image quality insufficient for diagnostic purposes), sufficient (sufficient image quality for diagnostic purposes but noticeable artifacts or image-quality issues), good (only minor artifacts or image-quality issues), and excellent (no artifacts or image-quality issues). MS lesions were assessed by number and localization (periventricular, juxtacortical, infratentorial) and for the presence of black holes, defined as lesions with lower signal intensity than the normal-appearing white matter in T1WI (yes, no). Discrepancies were compared to the gold standard: the majority decision of the 2 original raters based on all available imaging data, including the 3D T1WI and FLAIR sequences, with any discrepancies resolved by a third rater, senior neuroradiologist (M.K.-W.). Due to the difficulty of quantifying and delineating confluent-versus-nonconfluent lesions for the lesion count, manual lesion segmentations were additionally performed on the $2 \mathrm{D}$ synthetic and conventional images by a resident in radiology (T.G.) in ITK-SNAP (www.itksnap.org). ${ }^{17}$

Contrast-to-noise ratio analyses were performed by manual tracing of 12 circular ROIs. Two were placed in the CSF (ante- rior horns of the lateral ventricles bilaterally), 6 in the GM (thalami, frontal cortex, occipital cortex bilaterally), and 4 in the WM (centrum semiovale bilaterally, genu and splenium), and the measurements were averaged for each tissue type. For patients with MS, an additional ROI was placed in the largest MS lesion. Care was taken to avoid regions with artifacts and tissue borders. The contrast-to-noise ratios were calculated by dividing the intensity difference of the tissues by the median SD of all 12 ROIs.

\section{Volumetry}

Volumetric measurements from synthetic MR imaging were obtained by using the automatic segmentation in SyMRI, Version 7.2 RC. Conventional volumetric measurements were obtained by using MPRAGE in FreeSurfer (http://surfer.nmr.mgh.harvard. edu), Statistical Parametric Mapping (SPM; http://www.fil.ion.ucl.ac.uk/spm/ software/spm12), and the FMRIB Software Library (FSL; http://www.fmrib. ox.ac.uk/fsl). ${ }^{18-20}$ Automatic lesion segmentation based on the conventional 3D images was performed in the Lesion Segmentation Toolbox (Technische Universität München, Munich, Germany) for SPM, ${ }^{21}$ and lesion filling and masking were performed in FSL and SPM to reduce the bias of MS lesions on GM segmentations. ${ }^{22}$ Segmentations were quality-controlled by a resident in radiology (T.G.), and segmentation parameters in FreeSurfer, FSL, SPM, and the Lesion Segmentation Toolbox were optimized to ensure accurate volumetric data. Initial FSL parameters were chosen on the basis of previous recommendations. ${ }^{23}$ Software versions, input, parameters, and processing times can be found in On-line Table 1.

The volumetric measurements of interest were the brain volume (BV), the white matter volume, the gray matter volume, and the intracranial volume (ICV). In the SyMRI software, lesion volume is not estimated, but there is a segmentation of the remaining intracranial content that is not recognized as WM, GM, or CSF. This segmentation class is henceforth referred to as "non-WM/GM/CSF" and contains not only MS lesions but also flow voids in larger blood vessels. The brain tissue measurements were further normalized to the ICV, resulting in the brain parenchymal fraction (BPF), white matter fraction (WMF), gray matter fraction (GMF), and nonWM/GM/CSF fraction. Examples of the synthetic segmentation output can be seen in Fig 1.

\section{Statistical Analysis}

Statistical analysis was performed in SPSS Statistics 23 (IBM, Armonk, New York). The Shapiro-Wilk normality test was used to investigate the normality of distribution. Group differences were studied by using the Wilcoxon signed rank test for 

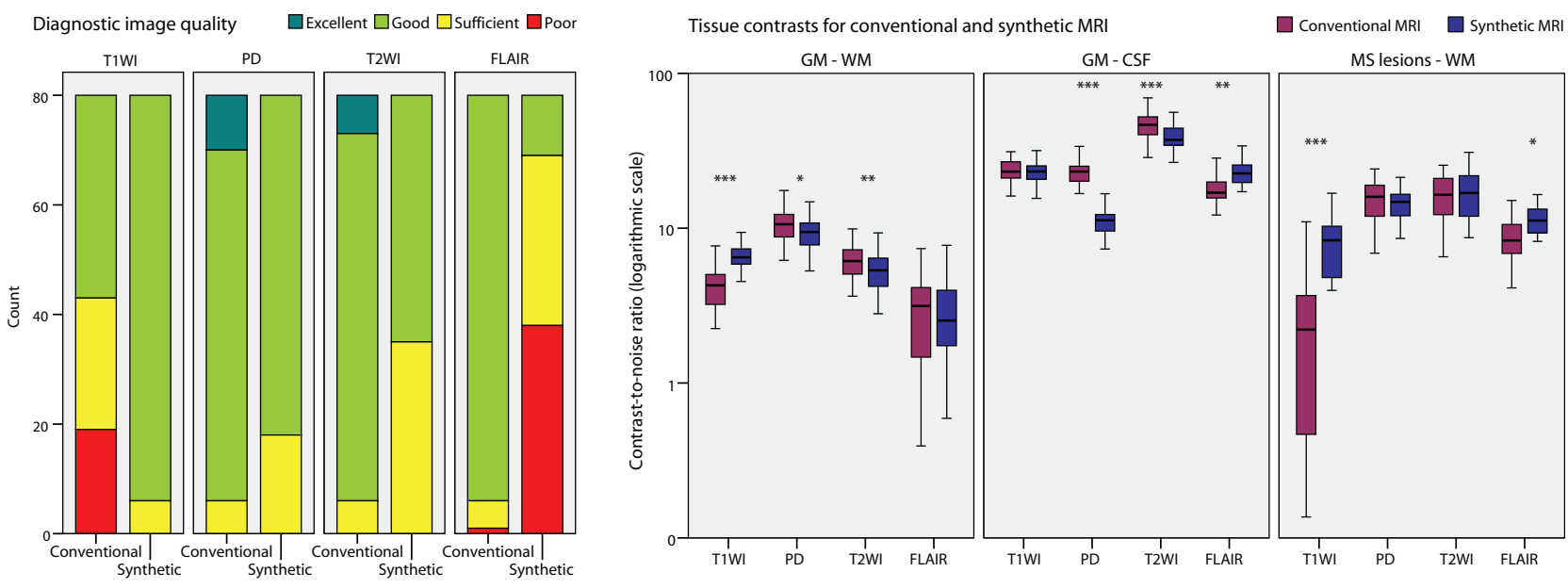

FIG 2. Comparison of the diagnostic image quality $(A)$ and contrast-to-noise ratios $(B)$ for conventional and synthetic MR imaging in patients with MS and controls. Wilcoxon signed rank test: single asterisk indicates $P<.05$; double asterisks, $P<.01$; triple asterisks, $P<.001$.

ordinal dependent data and the McNemar test for binary dependent data. The Spearman rank correlation coefficient was used for correlation analysis. Repeatability was quantified by using the intermeasurement coefficient of variance $(\mathrm{CoV})$ according to the following equation ( $A$ and $B$ being the first and second measurements per patient):

$$
\operatorname{CoV}=\frac{S D}{\text { Mean }}=\frac{\sqrt{\left(\frac{\sum(A-B)^{2}}{2 n}\right)}}{\frac{\sum(A+B)}{2 n}}
$$

Statistical significance was an $\alpha$ level of 5\% (2-tailed, equal variances not assumed).

\section{RESULTS}

\section{Image Quality and Diagnostic Accuracy}

A representative example of conventional and synthetic MR images is seen in Fig 1 . The diagnostic image quality and contrastto-noise ratio measurements are given in Fig 2. Synthetic images were of sufficient or good quality overall, except for FLAIR images, which were degraded by basilar artery and CSF pulsation artifacts in $48 \%$ of the scans. Other image artifacts encountered in the synthetic MR images were chemical-shift displacement, discrete Gibbs ringing phenomenon along the superior sagittal sinus, and a sinusoidal intensity difference in the CSF in PD-weighted and T2WI in the anteroposterior direction, which was more easily detected if the lateral ventricles were enlarged. Artifacts are exemplified in On-line Fig 1. A proportion (23\%) of the conventional spin-echo T1WIs were considered of poor diagnostic quality, mainly due to pulsation artifacts and low tissue contrast. Meanwhile, none of the synthetic T1WIs were rated of poor diagnostic quality, and synthetic T1WIs were assessed as having better image quality overall for diagnostic purposes $(P<.001)$. The difference in tissue contrast was independently corroborated by objective differences in contrast-to-noise ratios, also illustrated in Fig 2.

All 40 participants were correctly classified as patients/controls with both MR imaging techniques, and incidental findings were identical: unspecific white matter changes in healthy controls $(n=3)$, pineal cysts $(n=3)$, mucosal thickening in the

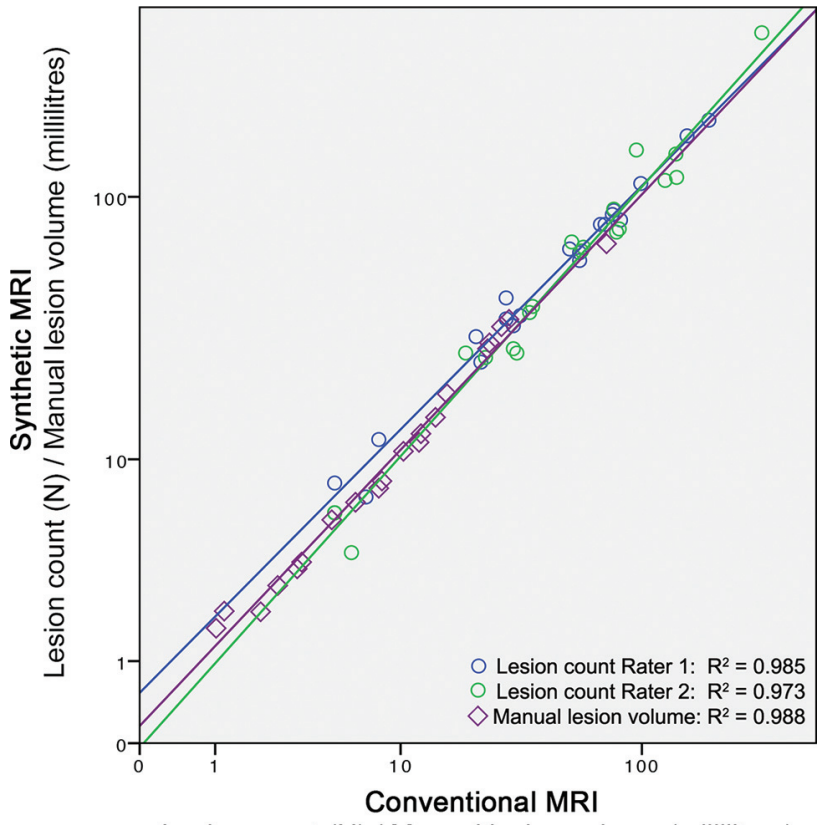

Lesion count $(\mathrm{N})$ / Manual lesion volume (millilitres)

FIG 3. MS lesion counts and manual MS lesion volume segmentation in conventional and synthetic MR imaging with linear regression lines.

maxillary sinuses $(n=2)$, cerebrovascular lesions $(n=1)$, contusional injury $(n=1)$, choroid plexus cyst $(n=1)$, arachnoid cyst $(n=1)$, and abnormally shaped eye bulb $(n=1)$. The lesion count was higher in synthetic MR imaging compared with conventional MR imaging (median, 71 versus 64 ; interquartile range, 62 versus $56 ; P<.001$ ), but this difference was on the same order of magnitude as the difference between the 2 raters (median, 74 versus 62; interquartile range, 68 versus 53), illustrated in Fig 3. Manual lesion segmentations corroborated this difference with higher manual lesion volumes in synthetic MR imaging ( 9.5 versus 9.3; interquartile range, 18 versus $16 \mathrm{~mL} ; P=.009$ ). The surplus in synthetic lesion volume was mainly due to inclusion of tissue considered "dirty-appearing" WM in the conventional images, exemplified by differences in manual lesion segmentations in On-line Fig 2. There was no statistical difference in the detec- 

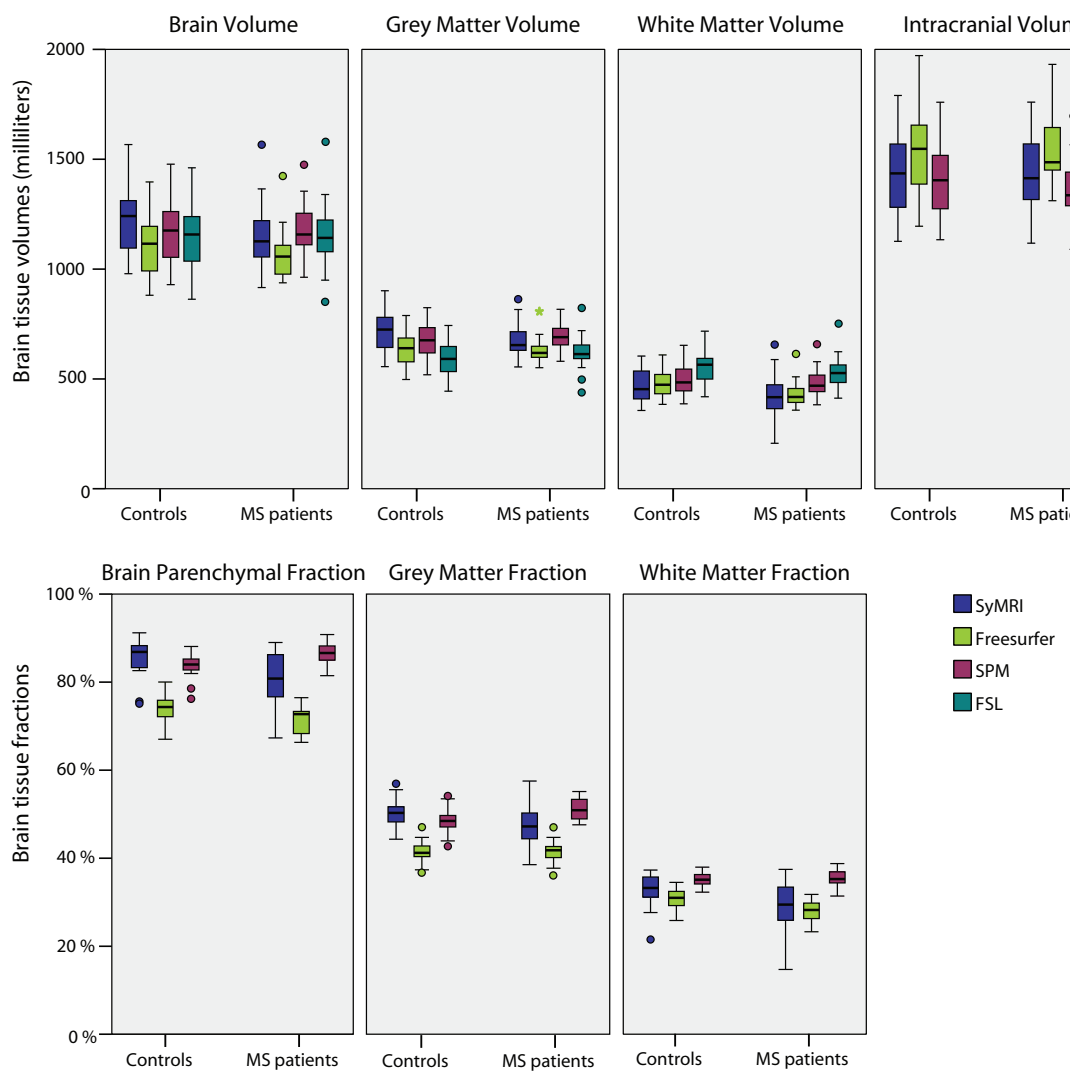

FIG 4. Comparison of conventional and synthetic MR imaging volumetry in patients with MS and controls.

tion of periventricular lesions $(P=.50,1$ false-negative finding on synthetic MR imaging), juxtacortical lesions ( $P=.50,1$ falsenegative finding on synthetic MR imaging), or infratentorial lesions ( $P=.77,1$ false-negative finding on conventional MR imaging, 1 false-positive finding on synthetic MR imaging). There was no statistical difference in the detection of black holes $(P=$ .50); 2 additional black holes were detected with synthetic MR imaging (1 false-negative finding on conventional MR imaging, 1 false-positive finding on synthetic MR imaging).

\section{Repeatability and Feasibility of Volumetry}

The SyMRI software provided volumetric measurements in the same order of magnitude as 3 commonly used volumetric software programs, illustrated in Fig 4. SyMRI was also the fastest segmentation method by several orders of magnitude (16 seconds compared with 12-187 minutes), without manual intervention, as reported in On-line Table 1. The test-retest repeatability of all volumetric methods is reported in Table 2. SyMRI had a lower repeatability error than FreeSurfer, FSL, and SPM for BV, ICV, BPF, and GMF. However, SyMRI had a similar error compared with the other volumetry methods regarding the segmentation of GM, WM, and WMF.

The SyMRI CoV for all 40 participants was $0.30 \%$ for BV and $0.23 \%$ for BPF, while the CoV was higher for WM and GM segmentations (gray matter volume and GMF, both $1.4 \%$; white matter volume, 1.8\%; WMF, 1.9\%). The largest repeatability errors in the SyMRI segmentations of patients with MS were for the smallest tissue volumes and fractions: the non-GM/WM/CSF vol-

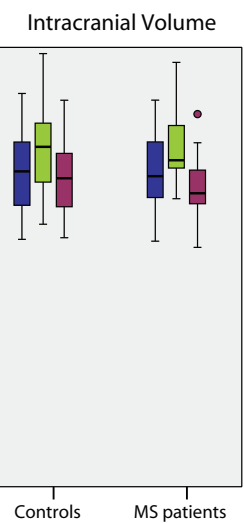

ume $(\mathrm{CoV}, 6.5 \%)$ and fraction $(\mathrm{CoV}$, $6.1 \%)$. The average absolute and relative measurement differences for patients with MS and controls are reported in On-line Table 2. In terms of the clinical significance, the Expanded Disability Status Scale score was correlated with SyMRI BPF $(r=-0.52, P=.02)$ and $\operatorname{GMF}(r=-0.53, P=.02)$, but not with WMF $(r=-0.2, P=.42)$ or non-GM/ WM/CSF fraction $(r=0.21, P=.37)$.

\section{DISCUSSION}

This is the first implementation of synthetic MR imaging on a Siemens MR imaging system and, to the best of our knowledge, the first independent evaluation of the diagnostic accuracy of the technique in MS. In this initial study, we found that synthetic MR imaging may be feasible as an alternative or complement to conventional PD-, T1-, and T2WI in MS. Differences in the contrast of lesions and dirtyappearing WM caused discrepancies in the lesion count and volume, but these differences did not affect the diagnostic classification or lesion topography. However, pulsation artifacts in the synthetic FLAIR imaging must be addressed before it can substitute for conventional FLAIR. Similar FLAIR artifacts have previously been reported in an implementation on a $1.5 \mathrm{~T}$ scanner from another manufacturer. ${ }^{11}$ Artifacts in the synthetic images could also be seen in the preprocessed DICOM files and were therefore not related to a postprocessing issue in the image reconstruction of the SyMRI software. Implementation of the sequence on the newer Siemens E11 software platform and on 1.5T scanners is underway, and we hope to re-evaluate these issues in the next implementation. Although incidental findings were identical with the $2 \mathrm{MR}$ imaging techniques, future studies will have to evaluate the diagnostic accuracy of synthetic MR imaging in pathologies other than MS. The time-savings compared with conventional MR imaging was 7\% for PD-, T1-, and T2WI (6:50 versus 7:22 minutes).

We have further shown that the volumetric measurements in the SyMRI are in agreement with those in other volumetric software and that SyMRI had the lowest repeat measurement errors for BV, ICV, BPF, and GMF among all tested volumetric methods. This finding is likely because the sequence inherently has information about $\mathrm{PD}$, the longitudinal and transverse relaxation rates, and $B_{1}$ field inhomogeneities, which can render more precise delineations of the intracranial and brain surfaces compared with just using information from T1WI. The multiparametric acquisition is equivalent to a multichannel approach, but without the need for image coregistration because the synthetic volumetrics are based on a single sequence. Because patients with MS are followed longitudinally, normalized brain volumetrics are of special interest. BPF was measured in patients with MS with SyMRI with repeatability errors (CoV, $0.25 \%$; average measurement difference, $0.06 \%$ ) well below the reported annual brain atrophy rate 
Table 2: Coefficients of variance of volumetric MRI measurements ${ }^{\mathrm{a}}$

\begin{tabular}{lccccccc}
\hline & $\begin{array}{c}\text { SyMRI } \\
\text { (40 Participants) }\end{array}$ & $\begin{array}{c}\text { SyMRI } \\
\text { (20 Patients with MS) }\end{array}$ & $\begin{array}{c}\text { SyMRI } \\
\text { (20 Controls) }\end{array}$ & $\begin{array}{c}\text { SyMRI } \\
\text { (5 Controls) }\end{array}$ & $\begin{array}{c}\text { SPM } \\
\text { (5 Controls) }\end{array}$ & $\begin{array}{c}\text { FreeSurfer } \\
\text { (5 Controls) }\end{array}$ & $\begin{array}{c}\text { FSL } \\
\text { (5 Controls) }\end{array}$ \\
\hline Brain volume & 0.30 & 0.35 & 0.25 & 0.14 & 0.29 & 0.21 & 0.18 \\
Gray matter volume & 1.4 & 1.2 & 1.6 & 0.64 & 0.81 & 0.78 & 0.25 \\
White matter volume & 1.8 & 1.6 & 2.0 & 0.81 & 0.68 & 0.70 & 0.28 \\
Intracranial volume & 0.26 & 0.28 & 0.24 & 0.12 & 0.17 & 0.15 & - \\
Brain parenchymal fraction & 0.23 & 0.25 & 0.21 & 0.14 & 0.17 & 0.20 & - \\
Gray matter fraction & 1.4 & 1.2 & 1.6 & 0.56 & 0.78 & 0.64 & - \\
White matter fraction & 1.9 & 1.7 & 2.1 & 0.96 & 0.79 & 0.67 & - \\
\hline
\end{tabular}

${ }^{a}$ All values represent the CoV in percentages. Volume is expressed in milliliters.

$(0.5 \%-1.3 \%) .^{24-27}$ The variability in measurements was larger in WMF and GMF (CoV, $1.2 \%$ and $1.7 \%$; average measurement difference, $0.4 \%$ and $0.5 \%$ ), but still lower than or equal to reported atrophy rates. The segmentation of the non-GM/WM/CSF fraction, which is the smallest tissue compartment, had the largest test-retest variability (CoV, 6.1\%; average measurement difference, $1.6 \%$ ) but may be of clinical importance because it is expected to partly reflect the lesion burden in MS. For comparison, MS lesion volume increases by approximately 5\%-10\% annually in MS. ${ }^{28,29}$ These results imply that GM, WM, and non-GM/WM/ CSF measurements should preferably be studied in perspectives longer than 1 year. From a clinical perspective, the SyMRI was the most practical segmentation method because it could be launched in the PACS and delivered robust volumetrics within a few tens of seconds. In radiologic practice, the feasibility of SyMRI volumetry lowers the threshold for being able to deliver quantitative biomarkers, which is becoming increasingly requested by our clinical colleagues.

This study has several strengths, such as the evaluation of both the diagnostic and volumetric output of SyMRI. The consecutive recruitment of the patients with MS is also advantageous because it reflects a typical panorama of patients with MS in a neurologic outpatient clinic, with all 3 subtypes of MS represented with a wide range of disease duration and Expanded Disability Status Scale scores, as well as the use of a control group. Limitations include a relatively small sample size and that repeat MPRAGE sequences could only be obtained in a subset of controls due to the length of the combined imaging protocol with reproducibility scans. In this study, we primarily harmonized settings in terms of voxel size and timing parameters, but unfortunately, the receiver bandwidth was not optimized in the same way. A lower bandwidth results in a higher signal-to-noise ratio and therefore hampers this comparison, but it also means that SyMRI was affected with chemical-shift displacement artifacts and theoretically longer minimum TEs and TRs, longer echo spacing, and increased susceptibility artifacts.

In terms of generalizability, the study is limited by the use of a 4-mm section thickness with a $30 \%$ gap, while a $3-\mathrm{mm}$ section thickness is recommended for MS studies with 2D sequences. ${ }^{30}$ Subsequently, we have been able to reduce this section thickness to $3 \mathrm{~mm}$, but the gap remains to reduce cross-talk between sections. Future technical effort should try to eliminate the need for this gap in SyMRI and should try to make high-resolution 3D acquisitions possible, which is increasingly important in MS studies. ${ }^{30,31}$ Previous studies of the effects of intravenous gadoliniumbased contrast media on the volumetric output of SyMRI indicate that it is important to consistently acquire synthetic MR imaging either pre- or postcontrast for comparability ${ }^{32}$; these findings are the reason we chose to focus on nonenhanced acquisitions in this study. Future studies will be able to clarify how synthetic MR imaging could be implemented as a postcontrast examination and in a longitudinal fashion in MS. Although the basic concepts of synthetic MR imaging implementation on other MR imaging systems are similar, a comparative study across 1.5 and $3 \mathrm{~T}$ scanners from the 3 major MR imaging systems is warranted. Lastly, an advantage with SyMRI that remains to be studied is the possibility of being able to adjust the synthetic TR, TE, and TI parameters post hoc, which may potentially allow the user to optimize the contrast between normal and pathologic tissues.

\section{CONCLUSIONS}

This study shows that synthetic MR imaging can be implemented on Siemens MR imaging scanners and can be an alternative to conventional MR imaging for generating diagnostic PD-, T1-, and T2-weighted images, but not yet FLAIR images, in patients with MS and healthy controls. Volumetric segmentations can be obtained with a few tens of seconds of processing without any extra scanning time in SyMRI. The measurements of BV and BPF have an excellent repeatability and are thus feasible for longitudinal studies in MS.

\section{ACKNOWLEDGMENTS}

We would like to thank the participants of the study and the staff at the Karolinska University Hospital MR imaging unit in Huddinge. We also acknowledge Stina Granberg, who has been helpful with graphic assistance. We further thank Karin Johnsson, Eric Westman, and Russell Ouelette for helpful comments on this article.

Disclosures: Tobias Granberg, Farouk Hashim, Yngve Forslin, Carmen Cananau, Sara Shams, Peter Aspelin, Maria Kristoffersen-Wiberg-RELATED: Grant: ALF Grant from Karolinska Institutet and Stockholm City Council.* Sten Fredrikson-UNRELATED: Payment for Lectures (including service on Speakers Bureaus): Allergan, Bayer, Biogen Idec, Genzyme, Merck, Sanofi, Teva; Payment for Development of Educational Presentations: Teva. *Money paid to the institution.

\section{REFERENCES}

1. Ma D, Gulani V, Seiberlich N, et al. Magnetic resonance fingerprinting. Nature 2013;495:187-92 CrossRef Medline

2. Riederer SJ, Suddarth SA, Bobman SA, et al. Automated MR image synthesis: feasibility studies. Radiology 1984;153:203-06 CrossRef Medline

3. Warntjes JB, Leinhard OD, West J, et al. Rapid magnetic resonance quantification on the brain: optimization for clinical usage. Magn Reson Med 2008;60:320-29 CrossRef Medline

4. Warntjes JB, Dahlqvist O, Lundberg P. Novel method for rapid, si- 
multaneous $\mathrm{T} 1, \mathrm{~T} 2{ }^{\star}$, and proton density quantification. Magn Reson Med 2007;57:528 -37 CrossRef Medline

5. Krauss W, Gunnarsson M, Andersson T, et al. Accuracy and reproducibility of a quantitative magnetic resonance imaging method for concurrent measurements of tissue relaxation times and proton density. Magn Reson Imaging 2015;33:584-91 CrossRef Medline

6. Ambarki K, Wåhlin A, Birgander R, et al. MR imaging of brain volumes: evaluation of a fully automatic software. AJNR Am J Neuroradiol 2011;32:408-12 CrossRef Medline

7. West J, Warntjes JB, Lundberg P. Novel whole brain segmentation and volume estimation using quantitative MRI. Eur Radiol 2012;22: 998-1007 CrossRef Medline

8. Ambarki K, Lindqvist T, Wåhlin A, et al. Evaluation of automatic measurement of the intracranial volume based on quantitative MR imaging. AJNR Am J Neuroradiol 2012;33:1951-56 CrossRef Medline

9. Vågberg M, Lindqvist T, Ambarki K, et al. Automated determination of brain parenchymal fraction in multiple sclerosis. AJNR Am J Neuroradiol 2013;34:498-504 CrossRef Medline

10. West J, Blystad I, Engström M, et al. Application of quantitative MRI for brain tissue segmentation at $1.5 \mathrm{~T}$ and $3.0 \mathrm{~T}$ field strengths. PLOS One 2013;8:e74795 CrossRef Medline

11. Blystad I, Warntjes JB, Smedby O, et al. Synthetic MRI of the brain in a clinical setting. Acta Radiol 2012;53:1158-63 CrossRef Medline

12. West J, Aalto A, Tisell A, et al. Normal appearing and diffusely abnormal white matter in patients with multiple sclerosis assessed with quantitative MR. PLoS One 2014;9:e95161 CrossRef Medline

13. Dua T; Rompani P; World Health Organization, Multiple Sclerosis International Federation. Atlas: Multiple Sclerosis Resources in the World, 2008. Geneva: World Health Organization; 2008

14. Goodin DS, Bates D. Treatment of early multiple sclerosis: the value of treatment initiation after a first clinical episode. Mult Scler 2009; 15:1175-82 CrossRef Medline

15. Bakshi R, Thompson AJ, Rocca MA, et al. MRI in multiple sclerosis: current status and future prospects. Lancet Neurol 2008;7:615-25 CrossRef Medline

16. Filippi M, Rocca MA. Multiple sclerosis: new measures to monitor the disease. Lancet Neurol 2013;12:12-13 CrossRef Medline

17. Yushkevich PA, Piven J, Hazlett HC, et al. User-guided 3D active contour segmentation of anatomical structures: significantly improved efficiency and reliability. Neuroimage 2006;31:1116-28 CrossRef Medline

18. Reuter M, Schmansky NJ, Rosas HD, et al. Within-subject template estimation for unbiased longitudinal image analysis. Neuroimage 2012;61:1402-18 CrossRef Medline

19. Jenkinson M, Beckmann CF, Behrens TE, et al. FSL. Neuroimage 2012;62:782-90 CrossRef Medline
20. Ashburner J, Friston KJ. Unified segmentation. Neuroimage 2005;26: 839-51 CrossRef Medline

21. Schmidt P, Gaser C, Arsic M, et al. An automated tool for detection of FLAIR-hyperintense white-matter lesions in multiple sclerosis. Neuroimage 2012;59:3774-83 CrossRef Medline

22. Vrenken H, Jenkinson M, Horsfield MA, et al; MAGNIMS Study Group. Recommendations to improve imaging and analysis of brain lesion load and atrophy in longitudinal studies of multiple sclerosis. J Neurol 2013;260:2458-71 CrossRef Medline

23. Popescu V, Battaglini M, Hoogstrate WS, et al; MAGNIMS Study Group. Optimizing parameter choice for FSL-Brain Extraction Tool (BET) on 3D T1 images in multiple sclerosis. Neuroimage 2012; 61:1484-94 CrossRef Medline

24. Miller DH, Barkhof F, Frank JA, et al. Measurement of atrophy in multiple sclerosis: pathological basis, methodological aspects and clinical relevance. Brain 2002;125:1676-95 CrossRef Medline

25. Simon JH. Brain atrophy in multiple sclerosis: what we know and would like to know. Mult Scler 2006;12:679-87 CrossRef Medline

26. Fotenos AF, Mintun MA, Snyder AZ, et al. Brain volume decline in aging: evidence for a relation between socioeconomic status, preclinical Alzheimer disease, and reserve. Arch Neurol 2008;65:113-20 Medline

27. Barkhof F, Calabresi PA, Miller DH, et al. Imaging outcomes for neuroprotection and repair in multiple sclerosis trials. Nat Rev Neurol 2009;5:256-66 CrossRef Medline

28. Molyneux PD, Filippi M, Barkhof F, et al. Correlations between monthly enhanced MRI lesion rate and changes in T2 lesion volume in multiple sclerosis. Ann Neurol 1998;43:332-39 CrossRef Medline

29. Fisniku LK, Brex PA, Altmann DR, et al. Disability and T2 MRI lesions: a 20-year follow-up of patients with relapse onset of multiple sclerosis. Brain 2008;131:808-17 CrossRef Medline

30. MAGNIMS study group. Evidence-based guidelines: MAGNIMS consensus guidelines on the use of MRI in multiple sclerosisestablishing disease prognosis and monitoring patients. Nat Rev Neurol 2015;11:597-606 CrossRef Medline

31. Traboulsee A, Simon JH, Stone L, et al. Revised Recommendations of the Consortium of MS Centers Task Force for a Standardized MRI Protocol and Clinical Guidelines for the Diagnosis and Follow-Up of Multiple Sclerosis. AJNR Am J Neuroradiol 2015 Nov 12. [Epub ahead of print]

32. Warntjes JB, Tisell A, Landtblom AM, et al. Effects of gadolinium contrast agent administration on automatic brain tissue classification of patients with multiple sclerosis. AJNR Am J Neuroradiol 2014;35:1330-36 CrossRef Medline 